\title{
Three Patterns Programmable Russian Form Functional Electrical Stimulator
}

\author{
Abbas Orand ${ }^{1}$, Genichi Tanino ${ }^{2}$, Hiroyuki Miyasak $^{3}$, Kotaro Takeda ${ }^{4}$, Shigeru Sonoda $^{5}$ \\ ${ }_{1,2,3,4}$ Fujita Memorial Nanakuri Institute, Fujita Health University, Japan \\ ${ }^{5}$ Department of Rehabilitation Medicine II, School of Medicine, Fujita Health University, Japan
}

\begin{tabular}{|c|c|}
\hline Article Info & ABSTRACT \\
\hline Article history: & \multirow{9}{*}{$\begin{array}{l}\text { In this paper, a programmable, multi-pattern, wide frequency and duty cycle } \\
\text { range electrical stimulator is presented. Using a programmable micro- } \\
\text { controller, two waves of carrier and modulating sources are produced. By } \\
\text { modulating the two sources, } 3 \text { bi-phasic charge-balanced rectangular, } \\
\text { triangular and sinusoidal stimulating patterns are produced. The frequency } \\
\text { range of the carrier is fixed at } 2.5 \mathrm{kHz} \text { and the carrier source frequency can } \\
\text { be adjusted between } 1 \text { and } 500 \mathrm{~Hz} \text {. The duty cycle of both sources can be } \\
\text { adjusted between } 10 \% \text { and } 90 \% \text {. } \\
\text { Copyright } \odot 2016 \text { Institute of Advanced Engineering and Science. } \\
\text { All rights reserved. }\end{array}$} \\
\hline Received Jun 11, 2016 & \\
\hline Revised Aug 22, 2016 & \\
\hline Accepted Sep 6, 2016 & \\
\hline Keyword: & \\
\hline \multirow{4}{*}{$\begin{array}{l}\text { Electrical and electroni } \\
\text { Electrical stimulation } \\
\text { Instrumentation }\end{array}$} & \\
\hline & \\
\hline & \\
\hline & \\
\hline \multicolumn{2}{|l|}{ Corresponding Author: } \\
\hline \\
\hline \multicolumn{2}{|c|}{ Division of Rehabilitation, } \\
\hline \multicolumn{2}{|c|}{ Fujita Health University, } \\
\hline \multicolumn{2}{|c|}{ Tsu, Mie 514-1296, Tel: +81 059-252-2744, Fax: +81 059-252-0710, Japan. } \\
\hline \multicolumn{2}{|c|}{ Email: orand@fujita-hu.ac.jp } \\
\hline
\end{tabular}

\section{INTRODUCTION}

Electrical stimulators are used for the rehabilitation of patients [1] and for research [2-3]. One method for producing an electrical stimulation is current-mode in which a constant current over a specific time is applied to a bodily part and the other method is voltage-mode in which a constant voltage is applied.

Electrical stimulators are designed and developed in previous studies [2], [4-13]. A microcontroller is used for the generation of a electrical stimulation in [2], [4-13]. The convenience of micro-contrllers have made these devices also popular in other applications [14-15].

All of the developed electrical stimulators are unique and present one or several forms of electrical stimulaitons. However, none of the before mentioned researches apply the concept of generating two signals, carrier and modulating sources, with the same micro-controller for the generaiton of Russian form electrical stimulation. The objective of this research was to present a new method for the generation of 3 rectangular, triangular and sinusoidal stimulation patterns in Russian form. We show how it is feasible, simple and extendable to use a micro-controller to generate these 3 patterns of electrical stimulaitons.

\section{RESEARCH METHOD}

The architecture of the functional electrical stimulator is shown in Figure 1. The power source is isolated from the stimulating unit using galvanic medical power supply and DC-DC converters. The stimulating part consists of modulating (AD835), high pass filter (HPF) and low pass filter (LPF) sections and amplification units. For linearity and instant output response, buffers are added before and after HPF and LPF secions. 


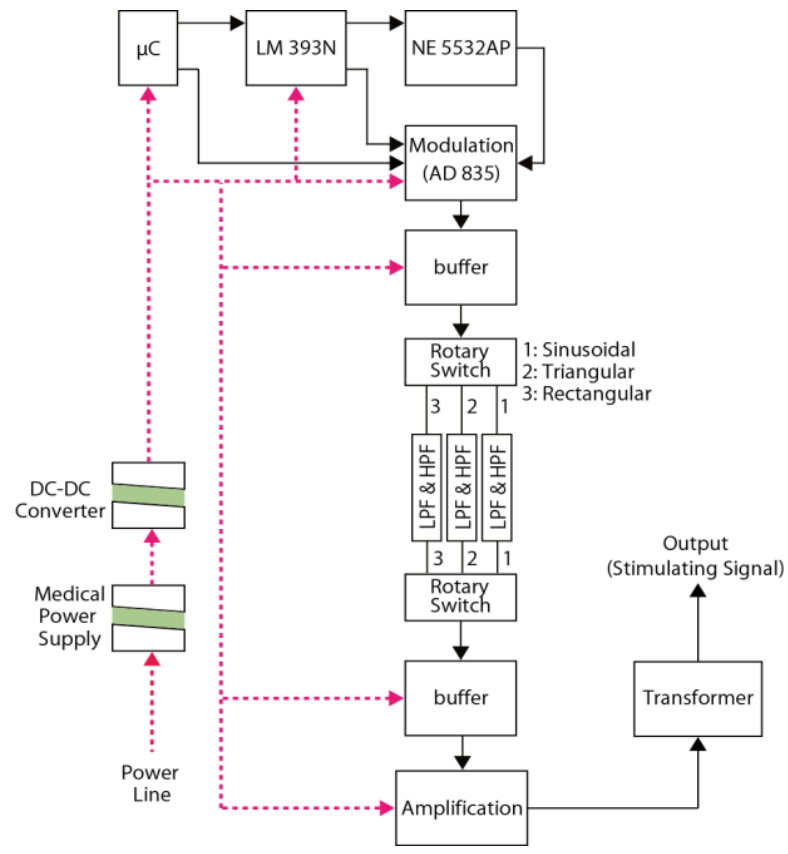

Figure 1. Functional Electrical Stimulator Architecture is Shown. HPF: High Pass Filter, LPF: Low Pass Filter

The carrier source was set to $2.5 \mathrm{kHz}$ [16]. The modulating source can be changed between 1 and $500 \mathrm{~Hz}$. The duty cycle of both the carrier and modulating sources can be adjusted between 10 and 90 percent.

\subsection{Modulation Section}

The amplitude modulation unit was used because of the need for the Russian forms of electrical stimulations of rectangular, triangular and sinusoidal wave patterns. The high input impedance of the unit makes signal source loading negligible while the unit's low output impedance or in other words its high output capability allows low impedance loads to be driven.

The generation of stimulating signal is based on the modulation of 2 sources, $\mathrm{X}$ (modulating) and $\mathrm{Y}$ (carrier) (Figure 2). The two signals $\mathrm{X}$ and $\mathrm{Y}$ are generated using an ARDUINO UNO (Arduino Co.) micro-controller. Their amplitudes are adjusted in such a way that the amplitude of $Y$ be half of that of X. A $\mathrm{HPF}$ is used to remove the dc offset and for centering Y. By using a 4-quadrant multiplier AD835, X and Y sources are modulated as the output, $W_{\text {output }}$.

$$
W_{\text {output }}=\mathrm{XY} / \mathrm{U}+\mathrm{Z}
$$

where $U$ and $Z$ are 1 and 0 volts, respectively. Therefore,

$$
W_{\text {output }}=\mathrm{XY}
$$




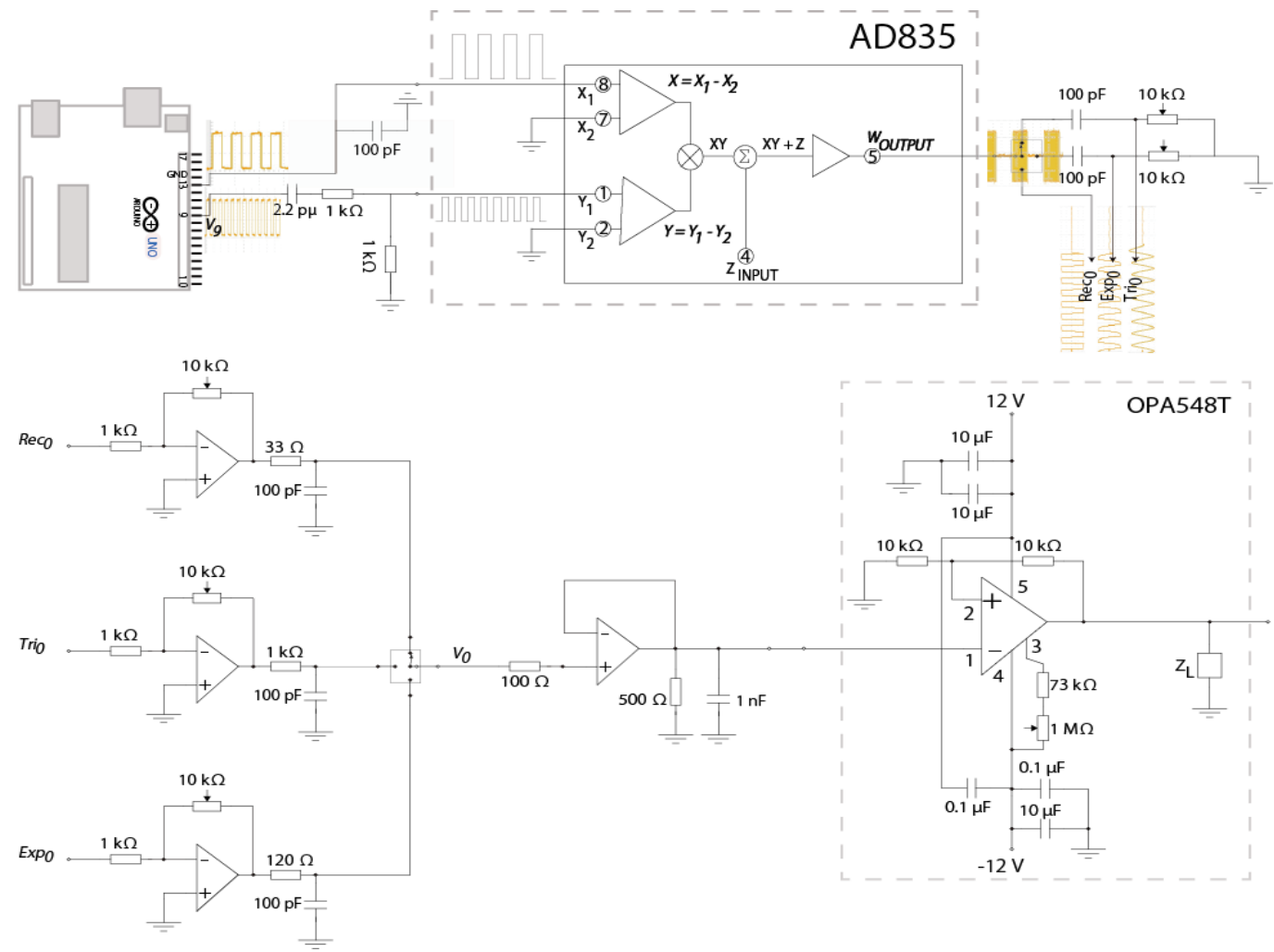

Figure 2. Electrical Stimulator Circuitry is shown. It Consists of Modulation Section (AD835), HPF Section, LPF and Amplitude Adjustment Sections, Buffering, Amplification and Current Limiting Sections

\subsection{HPF and LPF Sections}

The carrier source of the stimulating signal was chosen as $2.5 \mathrm{kHz}$. The HPF was used to remove the offset voltages and to convert the rectangular waves into waves of triangular and sinusoidal forms. The amplification and LPF sections were used for adjusting the amplitude and form of the waves of the 3 types of stimulating patterns.

\subsubsection{Amplification and Current Limiting}

In order to provide the inductor $\left(Z_{L}\right)$ with stable amplitude and to limit the current of the stimulating wave between 0 and $300 \mathrm{~mA}$, the output of the HPF and LPF $\left(V_{0}\right)$ were passed through a buffer and an amplification section.

\section{RESULTS}

The plots of 5 and $500 \mathrm{~Hz}$ rectangular source waves and their respective patterns are shown in Figure 3. Plots of 3 rectangular, triangular and sinusoidal source waves of $20 \mathrm{~Hz}$ and the pattern of a $20 \mathrm{~Hz}$ triangular source are shown in Figure 4. The carrier source of all 3 stimulating patterns was $2.5 \mathrm{kHz}$. 

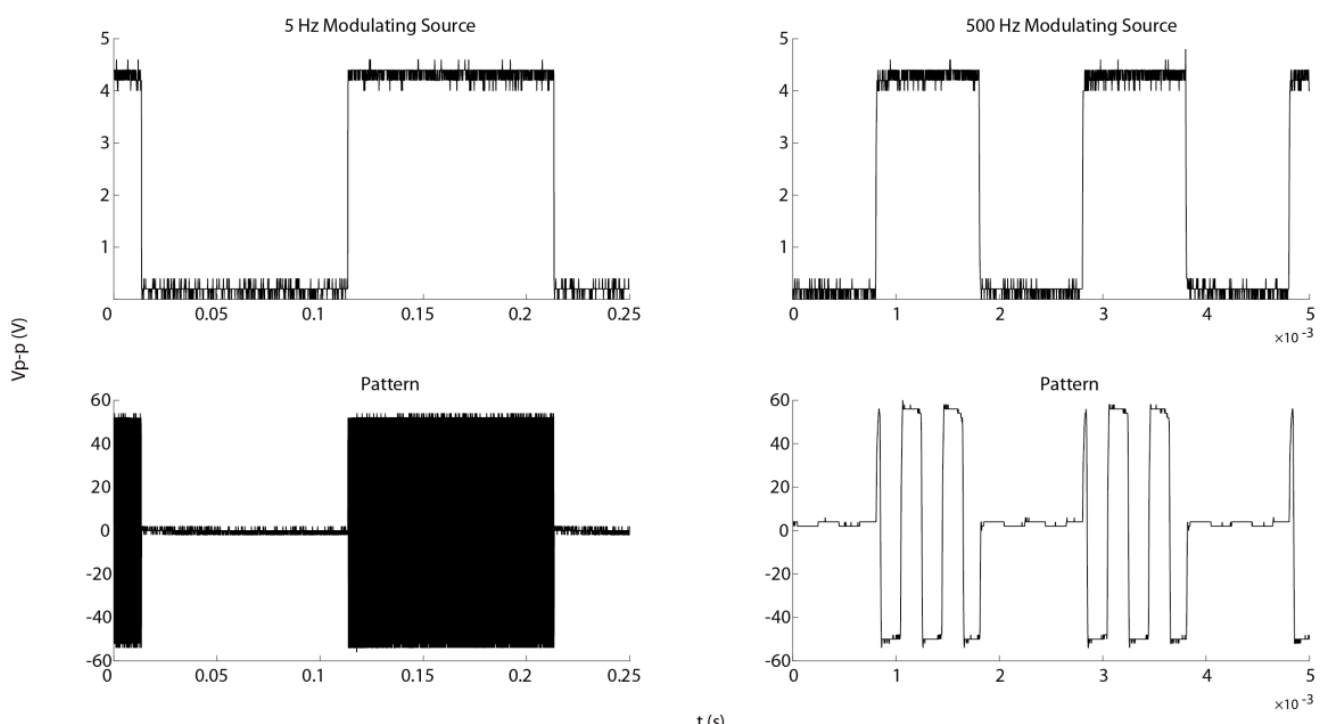

Figure 3. The modulating sources of frequencies of 5 and $500 \mathrm{~Hz}$ and their respective stimulating patterns for a rectangular waveform are shown. The duty cycles of both modulating and carrier sources are $50 \%$
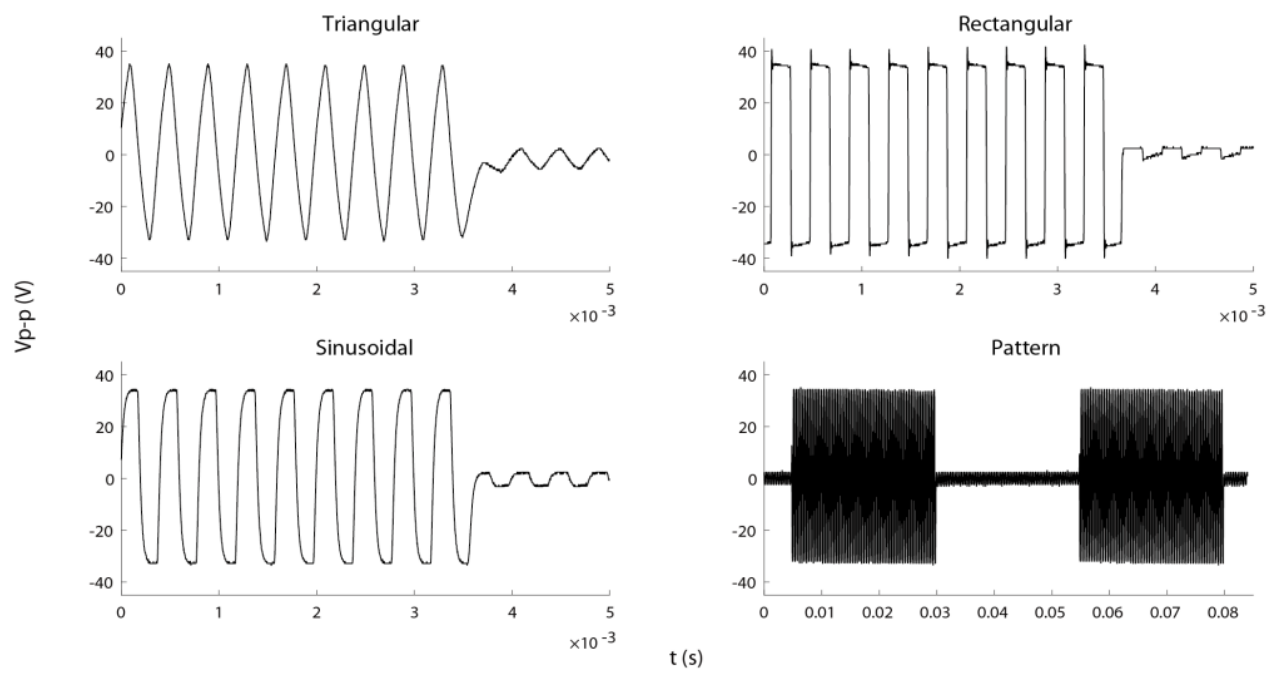

Figure 4. Three stimulating carrier sources of triangular, rectangular, and sinusoidal together with the pattern of a $20 \mathrm{~Hz}$ modulating triangular source are shown. The duty cycles of both modulating and carrier sources are $50 \%$

\section{DISCUSSION}

A new form of electrical stimulation, Russian, was popular amongst athelets [16]. In order to investigate the effect of such alternating electrical stiumulaiton, comparison to other wave forms were made in these researches [17-18]. Other forms of electrical stimulaitons have also been developed [2], [4-13]. In terms of charge, phase, and form, the developed electrical stimulations can be classified as it is shown in Table 1. Triangular and quasi-trapezoidal stimulating waves are also designed and developed in this research [12].

A microprocessor is used in this study [13] to produce 26 fixed and programmable protocols of treatments (eg. traebert, diadynamic, exponential, Russian, etc). Rectangular and triangular waveforms are not amongst the programmable or fixed protocols and it is not mentioned whether they have applied the modulation of waves priniciple for the generation of Russian form of stimulating waves. In these studies, [2], [4-5], [7], [9], [11-12] micro-controllers are also used. It is just in this work [9], that rectangular and triangular wave forms are generated amongst 6 distinct wave forms. 
Table 1. Characteristics of previously designed electrical stimulators in terms of charge, form and phase of the stimulating waves

\begin{tabular}{|c|c|c|c|c|c|c|c|c|}
\hline \multirow{2}{*}{$\begin{array}{l}\text { Previous } \\
\text { Researches }\end{array}$} & \multicolumn{2}{|c|}{ Charge } & \multicolumn{4}{|c|}{ Wave Form } & \multicolumn{2}{|c|}{ Phase } \\
\hline & balanced & not-balanced & Rec & Sin & Exp & Spike & mono-phasic & bi-phasic \\
\hline [2] & - & $\checkmark$ & $\checkmark$ & - & - & - & $\checkmark$ & - \\
\hline [4] & $\checkmark$ & $\checkmark$ & - & - & - & - & $\checkmark$ & $\checkmark$ \\
\hline$[5]$ & - & $\checkmark$ & $\checkmark$ & - & - & - & - & $\checkmark$ \\
\hline [6] & $\checkmark$ & - & $\checkmark$ & - & - & - & - & $\checkmark$ \\
\hline [7] & $\checkmark$ & - & - & $\checkmark$ & - & - & - & $\checkmark$ \\
\hline [8] & - & $\checkmark$ & $\checkmark$ & - & - & - & $\checkmark$ & - \\
\hline [9] & $\checkmark$ & $\checkmark$ & $\checkmark$ & - & - & - & $\checkmark$ & - \\
\hline [10] & $\checkmark$ & - & $\checkmark$ & - & - & - & - & $\checkmark$ \\
\hline [11] & - & - & - & - & - & $\checkmark$ & $\checkmark$ & $\checkmark$ \\
\hline [12] & $\checkmark$ & - & $\checkmark$ & $\checkmark$ & $\checkmark$ & - & - & $\checkmark$ \\
\hline$[13]$ & - & - & - & - & $\checkmark$ & - & - & $\checkmark$ \\
\hline
\end{tabular}

Rec: Rectangular, Sin: Sinusoidal, Exp: Exponential

None of the developed devices mentioned earlier generate 3 rectangular, triangular and sinusoidal in a Russian form. To our knowledge, the concept of applying the carrier and modulating sources of a single micro-controller together with a modulator for generating 3 commonly used electrical wave forms in a Russian electrical stimulating pattern is new. One advantage that our designed device has in common to other methods also applying a microcontroller is the minimum number of components required that makes the final design compact. The $2^{\text {nd }}$ advantage of our design is its user interface capable extendability. This can be realized for example by adding an input port for reading the input signal. This input signal can be the electromyogram activity of a specific muscle such as tibialis anterior muscle. By modifying program of the microcontroller, the input signal can be used for triggering the electrical stimulation. Finally, the microcontroller provides the user with precise and large range of frequency and duty cycle generations options.

\section{CONCLUSION}

We applied 2 waves of carrier and modulating sources for the generation of 3 rectangular, triangular and sinusoidal waves in Russian stimulating patterns at different frequencies between 1 and $500 \mathrm{~Hz}$. The duty cycle of both the carrier and modulating sources can be adjusted between 10 and 90 percent. The carrier waveform is fixed at $2.5 \mathrm{kHz}$ which is one limitation of our developed functional electrical stimulator. This is due to the fact that we convert a rectangular waveform to triangular and sinusoidal ones using high pass filters. One solution to this can be the generation of both modulating and carrier waves in 3 rectangular, triangular and sinusoidal waves from the beginning in the micro-controller. Such modification of architecture of the functional electrical stimulator at the micro-controller also allows the generation of monophasic stimulating patterns which are preferred in some applications.

\section{REFERENCES}

[1] T. Bajd, et al., "Use of functional electrical stimulation in the lower extremities of incomplete spinal cord injured patients", Artif Organs, vol. 23, pp. 403-409, 1999.

[2] D. Ibrahim, et al., "Design of a low-cost microcontroller-based functional electronic stimulation device for drop foot correction", Instrumentation Science and Technology, vol. 41(6), pp. 556-573, 2013.

[3] T.M. Kesar TM, et al., "Functional electrical stimulation of ankle plantarflexor and dorsiflexor muscles. Effects on post-stroke gait", Stroke, vol. 40, pp. 3821-3827, 2009.

[4] L.M. Alonso, et al., "Design of a Transcutaneous Electrical Stimulator to Avoid the Postoperative Intestinal Effects", Proceedings of the 4th International Conference on Electrical and Electronics Engineering (ICEEE), pp. 90-93, Mexico City, September 2007.

[5] S. Farahmand, et al., "Wearable, Battery-Powered, Wireless, Programmable 8-Channel Neural Stimulator", Proceedings of the 34th IEEE Annual International Conference (EMGS), pp. 6120-6123, San Diego, August 2012.

[6] A. Masdar, et al., "Current Source with Low Voltage Controlled for Surface Electrical Stimulation", Proceedings of the 9th International Colloquium on Signal Processing and its Application (CSPA), pp. 161-164, Kuala Lumpur, March 2013.

[7] A. Miskon, et al., "2-Channel Defense Transcutaneous Electrical Stem Stimulator", Proceedings of the IEEE 
Conference on Biomedical Engineering and Sciences (IECBES), pp. 123-126, Kuala Lumpur, December 2014.

[8] S. Naumann, et al., "Dual-channel electrical stimulators for use by children with diplegic spastic cerebral palsy", Medical \& Biological \& Computing, vol. 23(5), pp. 435-444, 1985.

[9] E. Parede Martinez, "An Easy Wireless Multi-Waveform Urinary Electrical Stimulator "UES"”, Proceedings of the 5th International Conference on Electrical Engineering, Computing Science and Automatic Control (CCE), pp. 201-206, Mexico City, November 2008.

[10] R. Ranjandish, et al., "A Low-Power Digitally Closed-Loop Electrical Stimulator Suited for Low-Pulse-WidthStimulation", Proceedings of the 23rd Iranian Conference on Electrical Engineering (ICEE), pp. 1393-1397, Tehran, May 2015.

[11] S.D.H. Soedirdjo, et al., "Microcontroller-based Transcutaneous Electrical Nerve Stimulator with 8 Bit Cascade DAC", Proceedings of the International Conference on Instrumentation, Communications, Information Technology, and Biomedical Engineering (ICICI-BME), pp. 1-5, Bandung, November 2009.

[12] Q. Xu, et al., "programmable multi-channel stimulator for array electrodes in transcutaneous electrical stimulation", Proceedings of the IEEE/ICME International Conference on Complex Medical Engineering (CME), pp. 652-656, Harbin Heilongjiang, May 2011.

[13] Y. Forneiro, et al., "Therapeutic Electrical Stimulator", Proceedings of the 25th Annual International Conference of IEEE on Engineering in Medicine and Biology Society (IEMBS), vol. 2, pp. 1716-1719, Cancun, September 2003.

[14] T. Hussain, et al., "AVR Microcontroller Implementation for Customized Sound Generation", IJECE, vol. 2(1), pp. 112-119, 2012.

[15] A. Orand, et al., "On the Design and Development of a Zigbee-Based Multimodal Input-Output MonitoringActuating System", IJECE, vol. 5(5), pp. 1143-1152, 2015.

[16] A.R. Ward, et al., "Russian Electrical Stimulation: The Early Experiments", Physical Therapy, vol. 82(10), pp. 1019-1030, 2002.

[17] A. Aldayel, et al., "Comparison between alternating and pulsed current electrical muscle stimulation for muscle and systemic acute responses", J Appl Physiol, vol. 109, pp. 735-744, 2010.

[18] G. Kantor, et al., "The Effects of Selected Stimulus Waveforms on Pulse and Phase Characteristics at Sensory and Motor Thresholds", Physical Therapy, vol. 74(10), pp. 951-962, 1994.

\section{BIOGRAPHIES OF AUTHORS}
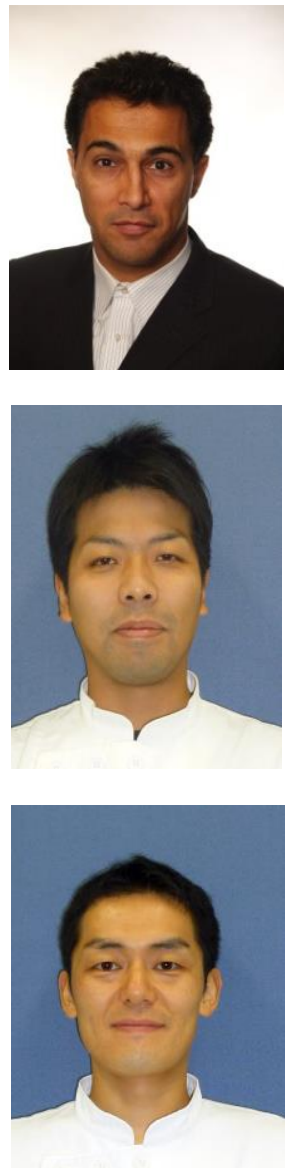

Abbas Orand obtained his B.Sc. and M. Sc. degrees in electrical and mechatronics engineerings from Dalhousie University in Canada and Siegen University in Germany in 2003 and 2008, respectively. From 2007 to 2008, he worked at Daimler AG in Germany as a researcher. He obtained his doctor of philosophy degree in the field of informatics and biosciences from Keio University in Japan in 2012. He is with the Rehabilitation Department of Fujita Memorial Nanakuri Institute at Fujita Health University since 2011. His fields of research interests are robotics, neuromuscular electrical stimulation, biomedical engineering and rehabilitation.

Genichi Tanino obtained his Bachelor of Arts and Master of Health Science degrees from the Open University of Japan and Osaka Prefecture Universities in 2006 and 2010, respectively. He is currently a fourth year Ph.D. student in the field of rehabilitation at the School of Medicine II of Fujita Health University. He has been working as an assistant professor at the Department of Rehabilitation of Fujita Memorial Nanakuri Institute, Fujita Health University. His research interests are physical therapy and rehabilitation.

Hiroyuki Miyasaka obtained his certification of occupational therapy in 1998. He received his Master of Arts and Ph.D. degrees from the Open and Fujita Health Universities in 2009 and 2013, respectively. Currently, he has been working as an assistant professor at the Rehabilitation Department of Fujita Memorial Nanakuri Institute of Fujita Health University in Japan. 


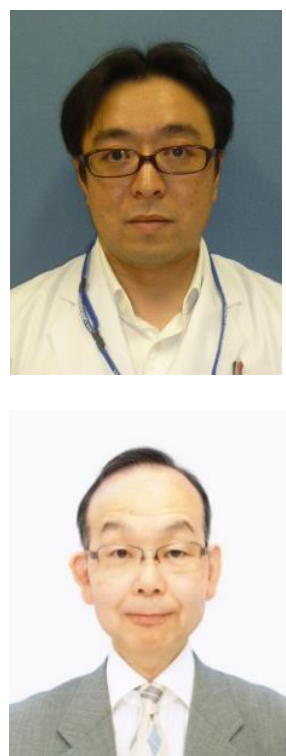

Kotaro Takeda was born in Japan in 1977. He graduated from the Faculty of Science and Technology, Keio University in 2000. In 2007, he received his Ph.D. degree of Science in Health Sciences from International University of Health and Welfare (IUHW). He worked as a Research Fellow at the Research Institute of National Rehabilitation Center for Persons with Disabilities from 2002 to 2005, as a CREST Researcher of Japan Science and Technology Agency at the IUHW Hospital from 2005 to 2009, as a Postdoctoral Research Fellow at the ATR Computational Neuroscience Laboratories from 2009 to 2011, and as a Chief Researcher at the National Hospital Organization Murayama Medical Center from 2011 to 2014. Since 2014, he has been an Associate Professor of the Fujita Memorial Nanakuri Institute, Fujita Health University, with research interests in engineering and motor physiology for stroke rehabilitation.

Sonoda Shiger received his Doctors of Medicine and of Philosophy from Keio University in 1985 and 1995, respectively. From 1997 to 2000, he was an assistant professor at the Department of Rehabilitation Medicine of the School of Medicine, Keio University. He worked respectively as an associate professor and a professor between the years of 2000-2002 and 2002-2007 at the Department of Rehabilitation Medicine of the School of Medicine of Fujita Health University. From 2003, he has served as the president of the Nanakuri Sanatorium of Fujita Health University. He has been the professor and chairman of the Department of Rehabilitation Medicine II of the School of Medicine of Fujita Health University since 2007. 